\title{
Erratum to: Non-surgical periodontal treatment of peri-implant diseases with the adjunctive use of diode laser: preliminary clinical study
}

\author{
Francesco Lerario $^{1} \cdot$ Marisa Roncati $^{3} \cdot$ Annalisa Gariffo $^{4} \cdot$ Enrica Attorresi $^{5}$. \\ Alessandra Lucchese ${ }^{6,7}$ - Alexandros Galanakis ${ }^{2}$ - Gaspare Palaia ${ }^{1}$ - Umberto Romeo ${ }^{1}$
}

Published online: 20 November 2015

(C) Springer-Verlag London 2015

\section{Erratum to: Lasers Med Sci}

DOI 10.1007/s10103-015-1785-7

The original version of this article, unfortunately, contained an error.

Alessandra Lucchese's affiliation should be changed to:

- Department of Orthodontics, Vita-Salute San Raffaele University, Milan, Italy

- Research Area in Dentofacial Orthopedics, Orthodontics and Pediatric Dentistry, Department of Dentistry, IRCCS San Raffaele Hospital, Milan, Italy

Her email address is lucchese.alessandra@hsr.it.

These affiliations and email are also reflected below.

The online version of the original article can be found at http://dx.doi.org/ 10.1007/s10103-015-1785-7.

\author{
Francesco Lerario \\ francescolerario@hotmail.it \\ Marisa Roncati \\ info@studioparmabenfenati.it \\ Annalisa Gariffo \\ annalisagariffo@libero.it \\ Enrica Attorresi \\ enrica.attorresi@libero.it \\ Alessandra Lucchese \\ lucchese.alessandra@hsr.it \\ Alexandros Galanakis \\ agalanakis@hotmail.it \\ Gaspare Palaia \\ gaspare.palaia@uniroma1.it
}

Umberto Romeo

umberto.romeo@uniroma1.it

1 Department of Oral and Maxillofacial Sciences, "Sapienza", University of Rome, Via Caserta 6, 00196 Rome, Italy

2 Division of Dentistry, Department of Pediatric Surgery, Bambino, Gesù Children's Hospital, Rome, Italy

3 "Sapienza" University of Rome, Rome, Italy

4 University of Palermo, Palermo, Italy

5 Marche Politecnic University, Ancona, Italy

6 Department of Orthodontics, Vita-Salute San Raffaele University, Milan, Italy

7 Research Area in Dentofacial Orthopedics, Orthodontics and Pediatric Dentistry, Department of Dentistry, IRCCS San Raffaele Hospital, Milan, Italy 